\title{
ARTICLE
}

\section{Neutron and gamma-ray source-term characterization of AmBe sources in Osaka University}

\author{
Isao Murata $^{\mathrm{a}^{*}}$, Iehito Tsuda ${ }^{\mathrm{a}}$, Ryotaro Nakamura ${ }^{\mathrm{a}}$, Shoko Nakayama ${ }^{\mathrm{b}}$, Masao Matsumoto ${ }^{\mathrm{b}}$ and Hiroyuki Miyamaru ${ }^{\mathrm{c}}$ \\ ${ }^{a}$ Graduate School of Engineering, Osaka University, 2-1 Yamada-oka, Suita, Osaka 565-0871, Japan; ${ }^{b}$ Graduate School of \\ Medicine , Osaka University, 1-7 Yamada-oka, Suita, Osaka 565-0871, Japan; ${ }^{c}$ Radiation Research Center, Osaka Prefecture \\ University, 1-1 Gakuen-cho, Nakaku, Sakai, Osaka 599-8531,Japan
}

Thermal / epi-thermal columns were constructed in the OKTAVIAN facility of Osaka University for basic studies of BNCT. For characterization of the columns, the spectrum and intensity of neutrons and the intensity of gamma-rays emitted from the AmBe sources used in the columns as a neutron source were measured. Neutrons were measured with the foil activation and multi-foil activation techniques, and gamma-rays were measured directly with an HpGe detector. The measured absolute intensity of neutrons is $2.4 \mathrm{E} 6 \mathrm{n} / \mathrm{sec}$. The measured neutron spectrum was fairly consistent with the previous result. The gamma-ray intensity was $1.8 \mathrm{E} 6$ photons/sec, which a little smaller than that of neutrons. The measured data are used for normalization of various experiments carried out for BNCT.

Keywords: BNCT; source term; AmBe; multi-foil activation; HpGe detector; $4.44 \mathrm{MeV}$

\section{Introduction}

Boron neutron capture therapy (BNCT) is known to be a very effective cancer therapy. In Japan, clinical tests are underway using nuclear reactors. BNCT can kill only tumors by charged-particles produced by ${ }^{10} \mathrm{~B}(\mathrm{n}, \alpha) \mathrm{L} \mathrm{Li}$ reaction. It is known that the effect to normal tissues could be suppressed substantially with BNCT.

However, there are problems recognized to be solved in BNCT. First, in Japan there exist only two nuclear reactor facilities available for BNCT. Accelerator based neutron sources (ABNS) are thus under investigation as a promising candidate for BNCT. At the moment, it is not yet realized because of various technical difficulties. The main reason is that the neutron intensity is weak and the energy is rather high. Patients should therefore be positioned very close to the target. As a result, the neutron spectrum would be strongly distorted and doses due to high energy neutrons and secondary gamma-rays become serious. The author's group is now developing a new device to characterize the neutron field with a position sensitive proportional counter for BNCT in ABNS as well as in nuclear reactors [1].

Also, a problem is known that it is difficult to know the exact BNCT dose in tumors. Currently, the irradiation time is determined with helps of simulation calculations and activation foils used during an irradiation. The key issue is that real-time monitoring of the BNCT dose is not possible. Our group is now

*Corresponding author. Email: murata@eei.eng.osaka-u.ac.jp studying a new detection device with CdTe crystals for developing a SPECT system to know three-dimensional BNCT dose distribution in real time [2].

For these researches for BNCT, small and easy-to-use thermal and epi-thermal neutron fields are indispensable. We have carried out design studies to construct these neutron fields for last few years [3]. As a neutron source, $\mathrm{AmBe}$ was used. Last year we constructed two graphite columns and replaced some parts of them with other material to make thermal and epi-thermal neutron fields. However, the specification of the AmBe sources, i.e., neutron spectrum and intensity, is not known except Am radioactivity, because the $\mathrm{AmBe}$ sources are originally used as a startup neutron source for a reactor subcritical assembly of Osaka University in the past. The objectives of the study are to clarify the neutron source spectrum and intensity and in addition the intensity of simultaneously emitted gamma-rays of $4.44 \mathrm{MeV}$.

\section{AmBe source}

In the OKTAVIAN facility of Osaka University, there are four AmBe sources, which were used as a neutron source for operating a reactor subcritical assembly of Osaka University. The intensity of each is 46.3 GBq. The AmBe source generates neutrons up to around $10 \mathrm{MeV}$ by the following nuclear reaction.

$$
{ }^{9} \mathrm{Be}+\mathrm{a} \rightarrow \mathrm{n}+{ }^{12} \mathrm{C}^{*}+\mathrm{Q}(5.704 \mathrm{MeV})
$$

a particles are emitted via a decay of ${ }^{241} \mathrm{Am}$. According to Geiger [4], 70 neutrons per $10^{6}$ a particles is reported. In reality, the a particle energy 
changes depending on the chemical form of $\mathrm{Am}$ and $\mathrm{Be}$, and how to mix both of them. Since the a energy change causes the neutron energy change, strictly speaking, it is necessary to measure the spectrum and intensity separately for each $\mathrm{AmBe}$ source stored in nuclear facilities.

The dimensions of the $\mathrm{AmBe}$ source are $3 \mathrm{~cm}$ in diameter by $5 \mathrm{~cm}$ in length. The source is double-sealed with stainless steel. Though the sources were quite old (fabricated in 1963), the intensity is almost the same as before because the half-life is as long as 400 years.

Also, from the excited residue nucleus, ${ }^{12} \mathrm{C}^{*}, 4.44$ $\mathrm{MeV}$ gamma-rays are emitted simultaneously. The $\mathrm{AmBe}$ can be used as a mono-energetic high energy gamma-ray source.

\section{Neutron measurement}

Neutron energy spectrum and intensity of the AmBe sources were measured with a multi activation foil technique. As a cross-check, the intensity was determined using a graphite thermal neutron column made with an AmBe source.

\subsection{Measurement with multi activation foils}

As is well known, neutron spectrum can be measured with a scintillation detector. However, in the case of $\mathrm{AmBe}$, neutron and gamma-ray discrimination is needed, which is a little complicated technique. In the present study, the multi activation foil technique was employed for the neutron spectrum measurement, considering that the foil activation method is quite simple and convenient, and as a result it can easily be applied to many facilities having such kind of strong AmBe sources.

\subsubsection{Selection of foils}

The dynamic range of neutron energy for foils to be selected is up to around $11 \mathrm{MeV}$ from the previous results. We examined threshold activation reactions up to $11 \mathrm{MeV}$. The criteria for the activation foil feature are set as follows: (1) Half-life of the foil should be longer than 5 minutes and shorter than 15 hours. (2) Evaluated cross section data for the activation foil should exist. (3) And, the number of counts more than several hundreds can be obtained by gamma-ray measurement for the period of half-life. As for the sample itself, the followings are taken into consideration: (1) Natural abundance of the nucleus to be activated should be more than $1 \%$. (2) Foils which cannot be obtained easily or are quite expensive should be avoided. (3) And, thin materials can be obtained by processing such that the correction factor due to thickness should be less than $20 \%$. From the above selection procedure, eight activation foils (nine reactions) were finally selected as shown in Table 1. Figure 1 describes each activation reaction cross section. Fortunately, threshold energies for the nine reactions are enough scattering in order to reproduce the neutron energy.
Table 1. Selected foils.

\begin{tabular}{llc}
\hline Nucleus & Reaction & $\begin{array}{c}\text { Effective } \\
\text { threshold (MeV) }\end{array}$ \\
\hline${ }^{113} \mathrm{In}$ & $\left(\mathrm{n}, \mathrm{n}^{\prime}\right)$ & 0.8 \\
${ }^{115} \mathrm{In}$ & $\left(\mathrm{n}, \mathrm{n}^{\prime}\right)$ & 0.8 \\
${ }^{111} \mathrm{Cd}$ & $\left(\mathrm{n}, \mathrm{n}^{\prime}\right)$ & 0.9 \\
${ }^{64} \mathrm{Zn}$ & $(\mathrm{n}, \mathrm{p})$ & 2.5 \\
${ }^{27} \mathrm{Al}$ & $(\mathrm{n}, \mathrm{p})$ & 4.2 \\
${ }^{56} \mathrm{Fe}$ & $(\mathrm{n}, \mathrm{p})$ & 6 \\
${ }^{24} \mathrm{Mg}$ & $(\mathrm{n}, \mathrm{p})$ & 6.4 \\
${ }^{27} \mathrm{Al}$ & $(\mathrm{n}, \boldsymbol{\alpha})$ & 7 \\
${ }^{59} \mathrm{Co}$ & $(\mathrm{n}, \alpha)$ & 7 \\
\hline
\end{tabular}

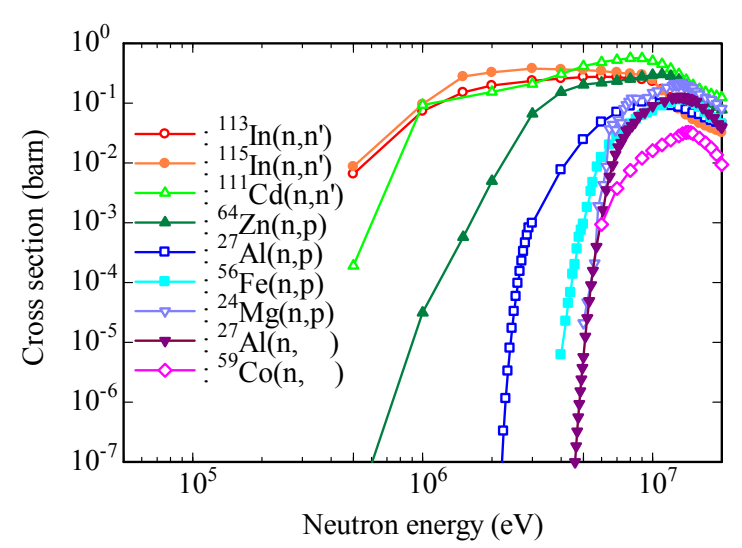

Figure 1. Cross sections of selected foils.

\subsubsection{Irradiation and measurement}

Experiments were conducted in "Large Experimental Room" of OKTAVIAN, which has a large experimental space of about $10 \times 10 \times 20 \mathrm{~m}^{3}$. The AmBe sources stored in the OKTAVIAN facility are rather massive, i.e., $3 \mathrm{~cm}$ in diameter and $5 \mathrm{~cm}$ in length. However, since double-sealed structure is employed, the part where Am and $\mathrm{Be}$ materials are packed is fairly small. By a collimation experiment, it was confirmed that the source could be dealt with as a point source. Activation foils are thus located at $5 \mathrm{~cm}$ distant from the $\mathrm{AmBe}$ source. A foil is set on an expanded polyethylene behind the source. After irradiation, cooling for an appropriate time, gamma-rays emitted from activated foils were measured with an HpGe detector. The measuring time is basically fixed to be one half-life. Thousands of counts were obtained except ${ }^{27} \mathrm{Mg}$ and ${ }^{56} \mathrm{Mn}$ created from aluminum and cobalt samples, respectively.

\subsubsection{Results}

Measured reaction rates for eight activation foils were unfolded by a process based on the Bayes' theorem with a response matrix for the foils evaluated from JENDL-Activation File [5]. The details of data processing are described elsewhere [6]. The result was compared with the previously measured spectrum [7] in Figure 2. It is noted that the cited reference spectrum is from ${ }^{239} \mathrm{Pu}-\mathrm{Be}$ and was bunched so that the energy bin becomes the same as the present measurement. The agreement is acceptable. However, because the number of energy bins is a little small so that the expected fine 
structure due to the nuclear levels of the target nucleus could not be confirmed. By integrating over energy, the source intensity can be estimated, which is $4.5 \times 10^{6} \mathrm{n} / \mathrm{sec}$. This value is for the case with the source casing. According to Geiger, the measured value is $3.2 \times 10^{6}$ $\mathrm{n} / \mathrm{sec}$, and $3.8 \times 10^{6} \mathrm{n} / \mathrm{sec}$ is reported as a theoretically calculated value. The present result shows a little larger compared to these previous results. It should be however noted that the spectrum disagreement might also be caused by the difference of the chemical property of Am and $\mathrm{Be}$.

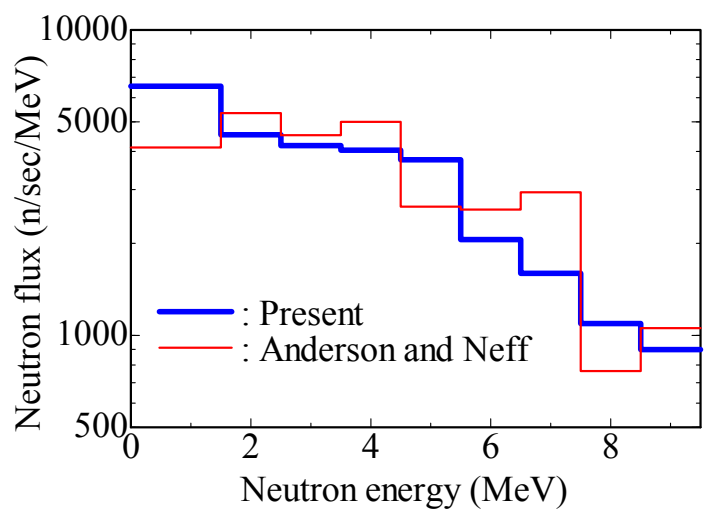

Figure 2. Measured neutron spectrum.

\subsection{Cross-check by integral experiment}

To check the disagreement with Geiger's value, an integral experiment with a graphite column was carried out. By this experiment the source intensity can be measured more accurately because one foil is enough in this kind of experiment and we can choose the one whose cross section accuracy is sufficiently high.

\subsubsection{Design and construction of graphite column}

At first, we designed a graphite column with MCNP5. An AmBe source was located in a cubic graphite column of $100 \times 100 \times 100 \mathrm{~cm}^{3}$. Changing the distance between the source and the sample from $5 \mathrm{~cm}$ to $25 \mathrm{~cm}$, the activation reaction rates of gold foils were calculated. As a result, the reaction rate shows its maximum at $5 \mathrm{~cm}$ and that of $15 \mathrm{~cm}$ shows a slight small value by $20 \%$. Taking into account the fact that placement error of the AmBe source can increase the error of the present estimation, the foil was set at $15 \mathrm{~cm}$.

\subsubsection{Irradiation and simulation calculation}

A graphite column was constructed in accordance with the design conducted in the previous section. An $\mathrm{AmBe}$ source and $\mathrm{Au}$ foil $\left(2 \times 2 \times 0.01 \mathrm{~cm}^{3}\right)$ were positioned and irradiation was performed for 3 days. After the irradiation, activity of the foil was measured with an HpGe detector. Also, transport calculations were carried out with a three dimensional model of the column including floor concrete by MCNP5. The source spectrum was assumed to be the one obtained in Sec. 3.1. With F4 tally, the reaction rate of ${ }^{197} \mathrm{Au}(\mathrm{n}, \boldsymbol{\gamma})$ was estimated.

\subsubsection{Results and discussion}

By comparing experiment and calculation, the neutron source intensity was derived as $2.4 \times 10^{6} \mathrm{n} / \mathrm{sec}$ so that the reaction rate of ${ }^{197} \mathrm{Au}(\mathrm{n}, \gamma)$ for both results became an equal value. This is the case without a casing. The obtained value is smaller than Geiger's and ours. Disagreement with Geiger is caused by the same reason mentioned in Sec. 3.1.3. The possible reason for the discrepancy within our results is thought to be uncertainty of the activation cross sections, i.e., all of the foils used in Sec. 3.1 have their evaluated activation reaction cross sections, however, some of them might not be validated sufficiently because they are not so frequently utilized. This may also be a cause of spectrum discrepancy discussed in Sec. 3.1.3. However, for the precise discussion for the spectrum, it is rather necessary to measure fine-energy-bin spectra by increasing the number of foils. The selection criteria in the present study were a little hard so that there are many foils which were abandoned. Contrary speaking, relaxing the criteria the number of foils can be increased. Also, direct measurements with a scintillation detector should be taken into account in the next step.

\section{Gamma-ray measurement}

From the AmBe source, $4.44 \mathrm{MeV}$ gamma-rays are emitted simultaneously. Intensities of both neutrons and gamma-rays are thought to be more-or-less the same. In this section, the intensity of gamma-rays was measured. Not only when using the AmBe source as a gamma-ray source, but also even in case of using it as a neutron source, the gamma-ray intensity is quite important information.

\subsection{Direct measurement with $\mathrm{Hp}-\mathrm{Ge}$ detector}

The measurement is quite simple. An AmBe source is placed in a large room and emitted gamma-rays are measured with an HpGe detector. The point is how to determine the detection efficiency for $4.44 \mathrm{MeV}$.

\subsubsection{Detection efficiency}

Detection efficiency for $\mathrm{HpGe}$ detector is easily fixed up to around $3 \mathrm{MeV}$ because suitable standard gamma-ray sources are available. In the present case, it is needed to determine the efficiency at $4.44 \mathrm{MeV}$. For this purpose, the graphite column constructed in Sec. 3.2 was used. An iron sample was set in the column and gamma-rays emitted via ${ }^{56} \mathrm{Fe}(\mathrm{n}, \gamma)$ reaction were measured. From an analysis with MCNP5, the optimum thickness of iron was $2 \mathrm{~cm}$ and it was found that the gamma-ray emission efficiency became the best when the iron sample was positioned on the surface of the column and the AmBe source was set just behind the iron sample. The relative intensities of emitted gamma-rays were cited from the BNL database [8]. Figure 3 shows the obtained efficiency curve. 


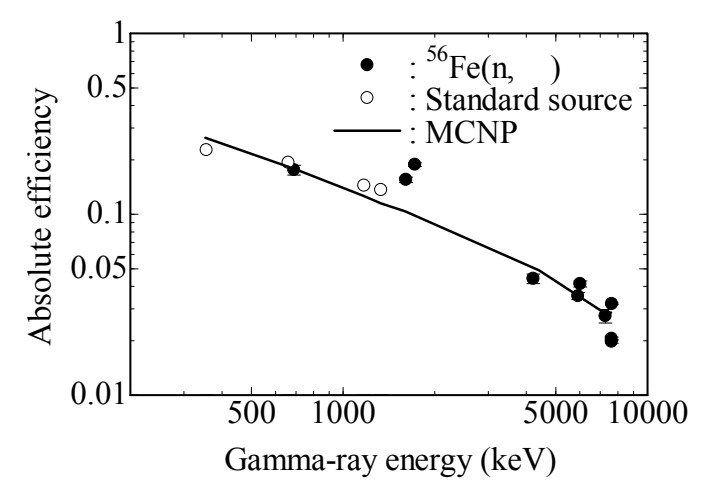

Figure 3. HpGe detector efficiency.

\subsubsection{Spectrum and intensity measurement}

Spectrum of gamma-rays emitted from the AmBe source was measured by the $\mathrm{HpGe}$ detector positioned at $3 \mathrm{~m}$ distant from the source. The measuring system was set close to the detector, but heavily shielded with $1 \mathrm{~m}$ thick iron wall. The intensity of $4.44 \mathrm{MeV}$ gamma-rays estimated from the net count of the photopeak was $1.8 \times 10^{6}$ photons $/ \mathrm{sec}$. This is a little smaller value than the neutron intensity of $2.4 \times 10^{6} \mathrm{n} / \mathrm{sec}$ for the case without a casing in Sec. 3.2.3.

\subsection{Correlation with the neutron intensity}

From the measured results, the intensity of gamma-rays shows $25 \%$ smaller than that of neutrons. The reason is discussed as follows: Figure 4 shows the level scheme of ${ }^{12} \mathrm{C}$. Now the $\alpha$ particle energy is 5.5 $\mathrm{MeV}$. The level of compound nucleus of ${ }^{13} \mathrm{C}$ is $\sim 11$ $\mathrm{MeV}$ higher than the ground state of the exit channel of ${ }^{12} \mathrm{C}+\mathrm{n}$. This means channels which should be taken into account are up to the five excited state of $10.8 \mathrm{MeV}$. Since gamma-rays of $4.44 \mathrm{MeV}$ are emitted by a transition from the first excited state to the ground state of ${ }^{12} \mathrm{C}$ and only $4.44 \mathrm{MeV}$ gamma-rays are measured, from other excited states a breakup reaction opens, which is a decay to three $\alpha$. According to JENDL-AN-2005 [9], cross sections for possible decay channels of $\left(\alpha, \mathrm{n}_{\mathrm{GS}}\right),\left(\alpha, \mathrm{n}_{1 \mathrm{st}}\right),\left(\alpha, \mathrm{n}_{2 \mathrm{nd}}\right)$ and $\left(\alpha, \mathrm{n}_{\text {continuum }}\right)$ are $50 \mathrm{mb}, 353 \mathrm{mb}, 39 \mathrm{mb}$ and $0 \mathrm{~b}$, respectively. For the channel of $\left(\alpha, \mathrm{n}_{1 \mathrm{st}}\right)$ emitting $4.44 \mathrm{MeV}$ gamma-rays, the cross section is about $80 \%$ out of the possible channels. In reality, $\alpha$ particles can induce nuclear reactions after slowing down. If the $\alpha$ particle energy is $2 \mathrm{MeV}$, the cross sections are going down and the ratio of the second reaction is about $75 \%$. Though it is difficult to estimate the exact value, this is consistent with the measurement.

\section{Conclusion}

Spectrum and intensity of neutrons and intensity of gamma-rays emitted from the AmBe sources stored in the OKTAVIAN facility of Osaka University were measured. The neutron spectrum was fairly consistent with the previous result. However, precise discussion was difficult because the number of energies was few. The absolute intensity was $2.4 \times 10^{6} \mathrm{n} / \mathrm{sec}$, which was a little smaller than the value obtained before. The gamma-ray intensity showed $25 \%$ smaller than that of neutron. In the next step, to estimate a finer-energy-bin neutron spectrum, we will carry out measurements with a scintillation detector, i.e., pulse height spectrum measurement, time-of-flight measurement and double scintillator measurement. The measured data will be used for various basic researches for BNCT in the future.

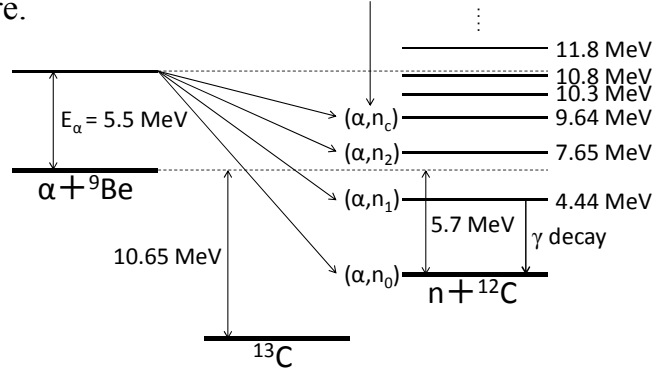

Figure 4. Energy level scheme of ${ }^{12} \mathrm{C}$.

\section{References}

[1] M. Ito and I. Murata, Thermal / epi-thermal neutron spectrometer with a ${ }^{3} \mathrm{He}$ position sensitive proportional counter, Proc. 2010 Annual Symposium on Nucl. Data, JAEA-Conf 2011-002, (2011), pp.95-100.

[2] S. Nakamura, T. Mukai, M. Manabe and I. Murata, Characterization Test of CdTe Detector Element Designed and Developed for BNCT-SPECT, Proc. of the 2011 Annual Symposium on Nucl. Data, JAEA-Conf 2012-001, (2012), pp.165-170.

[3] K. Harada and I. Murata, Design and Construction of A Thermal Column Using An Am-Be Neutron Source for BNCT studies, Proc. of the 2011 Annual Symposium on Nucl. Data, JAEA-Conf 2012-001, (2012), pp.129-134.

[4] K. W. Geiger and L. Van der Zwan, Nucl. Intrsum. Meth., 131, (1975), p.315.

[5] Y. Nakajima, JNDC WG on Activation Cross Section Data: JENDL Activation Cross Section File, Proc. the 1990 Symposium on Nuclear Data, JAERI-M 91-032, (1991), p.43.

[6] H. Kanasugi and I. Murata, Study on Neutron Spectrometer for Thermal to Epithermal Energy Regions with An Advanced Multi-Activation-Foil Method, Proc. of the 2011 Annual Symposium on Nucl. Data, JAEA-Conf 2012-001, (2012), pp.153-158.

[7] M. E. Anderson and R. A. Neff, Nucl. Intrsum. Meth., 99, (1972), p.231.

[8] http://www.nndc.bnl.gov/capgam/

[9] T. Murata, H. Matsunobu and K. Shibata: Evaluation of $(\alpha, \mathrm{xn})$ Reaction Data for JENDL/AN-2005, JAEA-Research 2006-052 (2006). 